\title{
Synchrotron x-ray microbeam diffraction from abalone shell
}

\author{
E. DiMasia) \\ Physics Department, Brookhaven National Laboratory, Upton, New York 11975 \\ M. Sarikaya \\ Materials Science and Engineering, University of Washington, Seattle, Washington 98195
}

(Received 10 October 2003; accepted 6 February 2004)

\begin{abstract}
Microstructured biomaterials such as mollusk shells receive much attention at present, due to the promise that advanced materials can be designed and synthesized with biomimetic techniques that take advantage of self-assembly and aqueous, ambient processing conditions. A satisfactory understanding of this process requires characterization of the microstructure not only in the mature biomaterial, but at the growth fronts where the control over crystal morphology and orientation is enacted. In this paper, we present synchrotron microbeam x-ray diffraction (XRD) and electron microscopy observations near the nacre-prismatic interface of red abalone shell. The relative orientations of calcite and aragonite grains exhibit some differences from the idealizations reported previously. Long calcite grains impinge the nacre-prismatic boundary at $45^{\circ}$ angles, suggestive of nucleation on (104) planes followed by growth along the $c$ axis. In the region within $100 \mu \mathrm{m}$ of the boundary, calcite and aragonite crystals lose their bulk orientational order, but we found no evidence for qualitative changes in long-range order such as ideal powder texture or an amorphous structure factor. XRD rocking curves determined the mosaic of calcite crystals in the prismatic region to be no broader than the $0.3^{\circ}$ resolution limit of the beamline's capillary optics, comparable to what can be measured on geological calcite single crystals.
\end{abstract}

\section{INTRODUCTION}

Molluscan mother-of-pearl, or nacre, exemplifies the capability of living organisms to produce high-strength and high-toughness mineralized tissues through biomolecular control of mineral crystals combined with an organic matrix. Through biomineralization processes, brittle materials such as calcium carbonates are incorporated into high-durability organic/inorganic laminates. ${ }^{1,2}$ Nacre is a calcium carbonate laminate material, and although the microstructure differs between species, structural studies remain of great interest in trying to further understand the growth processes. ${ }^{3-7}$ In the case of the red abalone, Haliotis rufescens, structural studies have been accompanied by experiments designed to elucidate the effects of proteins and biomolecules upon crystal growth and morphology. ${ }^{8-10}$ Despite these diverse efforts, the growth fronts and interfaces of the mineralized regions have yet to be characterized as completely as the mature material. For this reason, questions remain about the genesis of the material.

\footnotetext{
a) Address all correspondence to this author. e-mail: dimasi@bnl.gov

DOI: $10.1557 / J M R .2004 .0196$
}

There are two distinct mineralized regions in the abalone shell, illustrated schematically in Fig. 1(a), which depicts a cross section near the growth front at the edge of the shell. The outer layer is calcite, the stable $\mathrm{CaCO}_{3}$ polymorph, and referred to as the prismatic layer due to the $R 3 m$ (rhombohedral) symmetry of the calcite lattice. The prismatic layer, of thickness $0.5-3 \mathrm{~mm}$, provides hardness to the exterior of the shell. The calcite crystals have dimensions of order $5 \times 25 \mu \mathrm{m}$, and bulk x-ray studies indicate that the $c$ axis is preferentially oriented normal to the plane of the shell [M. Sarikaya, unpublished data]. Parallel to this prismatic layer is the motherof-pearl, or nacre. The nacreous layer of red abalone is assembled from flat platelets of the aragonite polymorph (Pnbc: orthorhombic) of $\mathrm{CaCO}_{3}$. These platelets have their [001] axes aligned normal to the interface and are stacked like bricks, "mortared" with a thin film of proteins and polysaccharides. ${ }^{1,2,6,11}$

Figure 1(b) shows a scanning electron micrograph of a cross section across the prismatic-nacre junction, illustrating the horizontal boundary plane, the $\geqslant 20 \mu \mathrm{m}$-scale calcite crystals, and the thin aragonite plates adjacent to them. Most of what is known about abalone shell formation comes from diffraction and microscopy of the natural material, or from cultivation of "flat pearls" grown on 


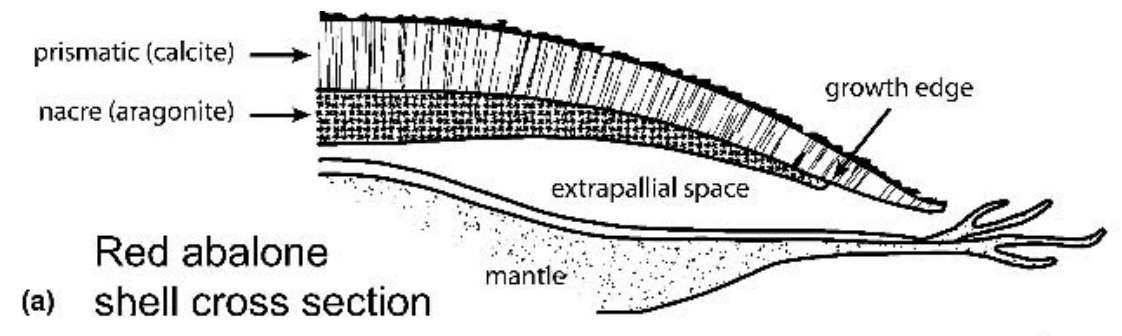

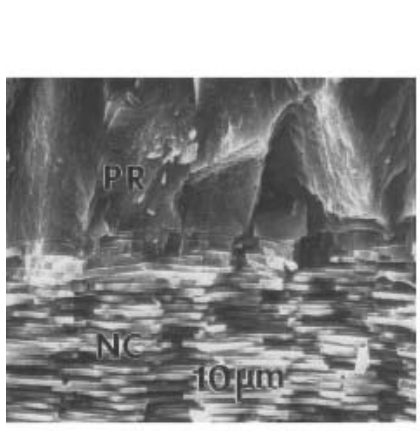

(b)

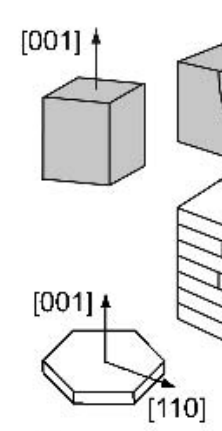

(c)

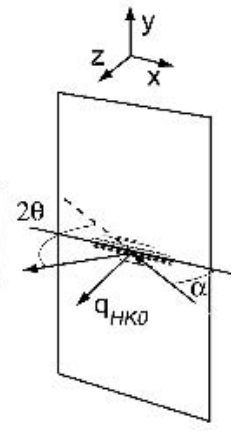

(d)

FIG. 1. (a) Red abalone shell cross section, showing the relationships between the mineralized layers and soft tissue in the vicinity of the growth edge, where new layers of nacre are deposited onto the prismatic layer making up the exterior of the shell. The vertical scale is exaggerated in the drawing. (b) SEM micrograph (secondary electron image) from fractured shell sample, showing prismatic (PR) and nacreous regions near the nacre-prismatic boundary. From Ref. 1. (c) Idealized depiction of the crystal alignment and morphology in the prismatic and nacre (NC) regions: calcite crystals with dimensions $\geqslant 20 \mu \mathrm{m}$, aligned with $c$ axis normal to the interface; and aragonite platelets $10 \mu \mathrm{m}$ across and $0.4 \mu \mathrm{m}$ thick, also with $c$ axis normal to the interface. (d) Scattering geometry for microdiffraction experiment from cut surface. The cut sample surface lies in the $x-y$ plane. The beam is incident on the sample at angle $\alpha$ and reflects at angle $2 \theta$ within the $x-z$ plane. The diffraction condition is met for grains having crystal planes parallel to the cut surface: according to the idealization in (c), these will be [HK0] reflections.

cover slips inserted against the animal's mantle. ${ }^{8,9}$ This knowledge has been distilled into idealized diagrams of the shell microstructure, as shown in Fig. 1(c), which identifies the orientation of crystals in each region. Of course, a comparison of Figs. 1(b) and 1(c) makes clear the differences between the planar idealizations and the actual crystals. Furthermore, the interface region between the two has not been well-characterized up to now. Yet this region is extremely important, as the material there may act to guide the formation and orientation of the aragonite crystals as the organism adds layers of nacre to its shell at the growth edge.

Electron diffraction has been the principal tool of structural studies up to now. The technique is appropriate for pinpointing the crystallinity and local orientation of small crystallites, but it can be difficult to determine the generality of the observations. As a complementary probe, x-ray scattering has notable advantages, such as good wavevector resolution and easily quantifiable intensities, that allow precise determinations of crystallinity and texture. However, bulk x-ray techniques actually do not characterize the material's microstructure very well, as it is organized on length scales ranging from the submicrometer thicknesses of the nacre platelets to $\geqslant 20 \mu \mathrm{m}$-scale calcite grains to the gross macroscopic curvature of the shell.

Synchrotron $\mathrm{x}$-ray microbeam diffraction provides a bridge between traditional $\mathrm{x}$-ray scattering and electron microscopy. With capillary optics, a spot size of order 1-20 $\mu \mathrm{m}$ can be focused onto a surface, and the diffracted peak positions and intensities measured with good resolution. The focus is preserved while translating the sample in submicrometer to millimeter steps, making it possible to map the locations of grains satisfying the diffraction condition for a given sample orientation. For hierarchically structured biomaterials, synchrotron x-ray microbeam diffraction is the best complementary probe to electron microscopy. In this paper we present microbeam diffraction data obtained at the National Synchrotron Light Source, in combination with electron microscopy observations, to describe the microstructure at the nacre-prismatic boundary in a cut section of the shell of Haliotis rufescens. We identify strings of oriented calcite grains of order $1 \mathrm{~mm}$ in length, oriented with their $[H K 0]$ axes in the boundary plane, and impinging the boundary at approximately $45^{\circ}$ angles. In the region within $100 \mu \mathrm{m}$ of the boundary, calcite and aragonite crystals lose their bulk orientational order relative to the layer plane. However, we find no evidence for extreme changes in long-range order such as ideal powder texture or an amorphous structure factor. X-ray diffraction (XRD) rocking curves were measured to characterize the crystal texture. Individual calcite crystals have resolution limited peak widths, indicating a crystal 
mosaic better than $0.3^{\circ}$. Our experiments provide the first structural mapping of individual crystallites across the nacre-prismatic boundary that spans the millimeter to micrometer length scales.

\section{EXPERIMENTAL}

Shell sections of red abalone collected from the west coast of Baja California, Mexico, were cut with a diamond saw to expose flat faces perpendicular to the nacre-prismatic boundary. These faces had dimensions of about $6 \times 18 \mathrm{~mm}$. The $\mathrm{x}$-ray studies were performed at a position within $500 \mu \mathrm{m}$ of the nacre-prismatic boundary and about $10 \mathrm{~mm}$ from the growth edge of the shell sample. [The scanning electron micrograph in Fig. 1(b) is from Ref. 11 and was performed on a fractured face of a different sample.]

Scattering experiments were performed on the IBM monochromatic microdiffraction instrument at the IBM/ MIT beamline X20A at the National Synchrotron Light Source at Brookhaven National Laboratory. This machine uses capillary optics to obtain focused spot sizes in the range $2-20 \mu \mathrm{m}$ and is built upon a vertical Huber two-circle diffractometer with partial chi and phi arcs, and a sample stage with $\pm 1 \mu \mathrm{m}$ resolution. ${ }^{12}$ The $\mathrm{x}$-ray wavelength $\lambda=1.459 \AA$. We used a reflection geometry as shown in Fig. 1(d). The cut shell surface lies in the $x-y$ plane, perpendicular to the nacre-prismatic boundary [this is the same as the front surface of the cut block depicted in Fig. 1(c)]. The x-rays reflect within the $x-z$ plane, and the illuminated spot can be positioned in the nacre, prismatic, or boundary regions by the sample translation stage moving in the $x$ and $y$ directions.

The $\mathrm{x}$-rays are focused onto the sample by refraction through a glass capillary. Because the optics must be brought as close as possible to the surface for the smallest beam size, the incident angle $\alpha$ of the x-ray beam onto the sample must be restricted to angles greater than $25^{\circ}$ or so. The beam footprint on this surface defines an illuminated area that is longer by about a factor of two along the beam path (defined as the $x$ direction in the sample plane) than the transverse ( $y$ direction) spot size. In our experiments, we estimate that at the closest working distance used, for $\alpha \approx 35^{\circ}$, the illuminated spot is approximately $5 \times 10 \mathrm{~mm}$. The beam divergence from the capillary, about $0.34^{\circ}$, broadens the angles over which crystal planes are aligned in the diffraction condition: Compared to standard high-resolution synchrotron diffraction beamlines, weak Bragg reflections from small grains are easier to identify, but the resolution for the rocking curve is worse by about a factor of 50 .

$\mathrm{X}$-rays are detected by a single channel scintillator detector (Bicron) at an angle designated by the label $2 \theta$, referenced to the direct incoming beam [dashed line in Fig. 1(d)]. The magnitude of the momentum transfer $q$ is a function of $2 \theta$, the scattering angle: $q=(4 \pi / \lambda) \sin (2 \theta / 2)$.
The diffraction peaks presented in this paper have $\alpha$ in the range 25-40 degrees, and $2 \theta$ ranges from 40 to 80 degrees, with minimal sample tilts in the transverse direction. This means that the momentum transfer vector (lying in the plane containing incident and reflected beam) can be considered to be contained in the $x-z$ plane. In most cases, the sample was measured with $\alpha \approx 2 \theta / 2$, that is, the momentum transfer vector $\mathbf{q}$ lies within a few degrees of the normal of the cut surface. Because the normal to the nacre-prismatic boundary itself is only well-defined to within a few degrees (not being ideally flat), we can regard these reflections as coming from crystal planes aligned parallel to the cut surface. The idealization of Fig. 1(c), where both calcite and aragonite $c$-axes lie along the $y$ direction, implies further that we should be sensitive only to $(H K 0)$ reflections in this geometry. We will show that this is approximately the case, except for regions quite near the nacre-prismatic boundary.

\section{RESULTS AND DISCUSSION}

\section{A. Grain mapping: Distribution of crystals near the boundary}

Our sample was positioned with the macroscopic (optical) nacre-prismatic boundary at $y=0$, and diffraction intensities at Bragg peak positions were explored to map out the nature of this boundary. At the Bragg peak positions, sample rocking curves were measured to assess the angular dispersion of the crystal domains. All calcite crystals exhibited rocking curves with widths of order $0.3^{\circ}$, which is the resolution limit of the divergent capillary optics of the beamline. Therefore, the prismatic crystal mosaic is no worse than $0.3^{\circ}$, and possibly comparable to geological single crystalline calcite (measured as a control).

After optimizing the sample orientation and scattering angle for each peak, intensities were mapped in the $x-y$ plane. Each color in Fig. 2 corresponds to a different sample alignment. The green lines are contours of intensity for $2 \theta=60.78^{\circ}, \alpha=35^{\circ}$, identified as the calcite (300) peak (nominal $2 \theta=60.83^{\circ}$ ). The green region thus delineates a millimeter-long calcite crystal (or collection of crystals aligned within $0.3^{\circ}$ of each other) lying with their $a$ axes within $4^{\circ}$ of the cut surface normal. The blue and red contours have $2 \theta=71.35^{\circ}$ and $71.52^{\circ}$, respectively. Black contours at the left of the figure (far in the calcite side of the sample) have $2 \theta=71.52^{\circ}$. All these scans were aligned with $\alpha=30^{\circ}-35^{\circ}$, so again, the diffracting planes are close to the surface plane. The nominal $2 \theta$ position of the calcite (220) is $71.55^{\circ}$. Therefore, the map of long colored grains in Fig. 2 shows calcite crystals lying with their $c$-axes close to parallel to the surface plane. The black contours in the aragonite side of the figure, $y>0$, are aligned with $2 \theta=71.95^{\circ}$. 


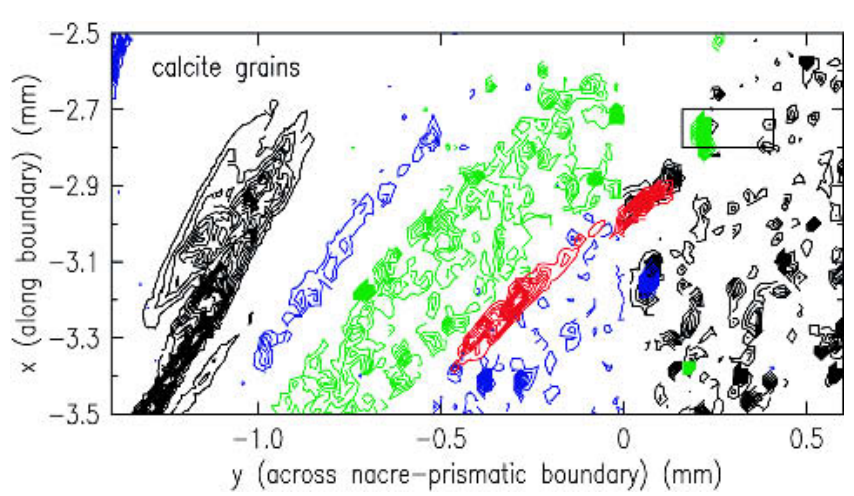

FIG. 2. Bragg peak intensity map in the prismatic $(x \leqslant 0.2)$ and nacre $(x \geqslant 0.2)$ regions of the sample surface. Long "grains" of like colors in the prismatic region correspond to calcite crystals with their $c$-axes lying within the surface plane (see text for detailed description). Black contours in the nacre region show the distribution of aragonite crystals oriented with the [400] axis along the surface normal. The region bounded by the rectangle is magnified in Fig. 3 .

We identify these as the aragonite (400) peak (nominal $2 \theta=72.03^{\circ}$ ). Note that the texture is different, with a smattering of intense spots rather than millimeter-long aligned regions. "Empty space" in the map simply shows regions for which no aligned crystal was identified in the current experiment.

These data provide a map of crystal orientation on length scales of millimeters, with approximately $10-\mu \mathrm{m}$ resolution, showing how the aligned calcite crystallites terminate near the nacre. The nacre-prismatic boundary is not flat, but is coarsely defined by the ends of the millimeter-long calcite rods. These grains run toward the boundary at approximately $45^{\circ}$ angles. The question remains whether the $c$ axis of the calcite points directly at the nacre-prismatic boundary or instead runs along the length of the grain. Limitations of the sample/spectrometer orientation in the current experiment prevent our answering this question directly here. Using bulk (nonmicrobeam) synchrotron XRD, we confirmed that $(00 L)$ reflections are found normal to the shell surface but distributed over an angular range of up to $10^{\circ}$ because of its macroscopic curvature; such bulk studies are not sensitive to the alignment of the nacre-prismatic boundary. Conversely, calcite crystals grown on cover glass inserted into red abalone mantle (flat pearls) have been reported to nucleate on (104) faces, but then grow along the $c$ axes. ${ }^{9}$ Micrographs of these samples show long calcite prisms sticking up from the flat substrate at $45^{\circ}$ angles, very suggestive of the data in Fig. 2. It was not known whether this slanted-crystal "flat pearl" growth mode occurs in natural shell. Our microdiffraction studies strongly suggest that it does.

\section{B. Fine scale map: Texture of aragonite}

Having characterized the nature of the boundary on the $100-\mu \mathrm{m}$ length scale, we investigated the boundary more closely: namely, looking for orientational correlations between the calcite closest to the boundary and the nearby aragonite crystals. Generally, in this boundary region, much smaller calcite crystals appear, separated from the dense aragonite regions by some tens of microns. Likewise, more "empty space" is observed around aragonite crystals of a given alignment. We chose the region bounded by the inset box in Fig. 2, close to a lone calcite crystal. A finer grain mapping was made, shown in Fig. 3. To avoid the difficulties in distinguishing the tails of calcite (220) and aragonite (400) peaks, which are close in $2 \theta$, we measured the intensities of the calcite (300) and aragonite (330) peaks, shown as green and blue contours, respectively. (Because the beam position was realigned between the two measurements, the $x$ and $y$ coordinates have shifted slightly between Figs. 2 and 3.)

The blue contours show the closest aragonite crystals that could be found near the calcite grain. These aragonite platelets are aligned with the (330) planes essentially parallel to the surface plane. The most intense scattering comes from regions $10-40 \mu \mathrm{m}$ across, just slightly larger than the illuminated spot size which is of order 5-15 $\mu \mathrm{m}$. There is no evidence for the persistence of grain alignment beyond this upper bound of $10-40 \mu \mathrm{m}$. Conversely, a lower bound on these orientational correlations has been determined from electron microscopy. Figure 4(a) shows a bright-field image of the stacked aragonite lamellae. The differences in contrast show that columns of stacked platelets have different crystallographic alignments with respect to their $a-b$ planes. The dark-field image of Fig. 4(b), corresponding to the selected-area diffraction pattern in Fig. 4(c), emphasizes that aligned regions generally extend at least $5-10 \mu \mathrm{m}$ (about 20 platelets) along the stacking direction.

The combined x-ray and electron studies demonstrate that along the stacking direction, aragonite tablets are aligned along the stack for $5-40 \mu \mathrm{m}$. This result can be put in the context of current speculations as to the mechanism by which successive nacre tablet layers attain their

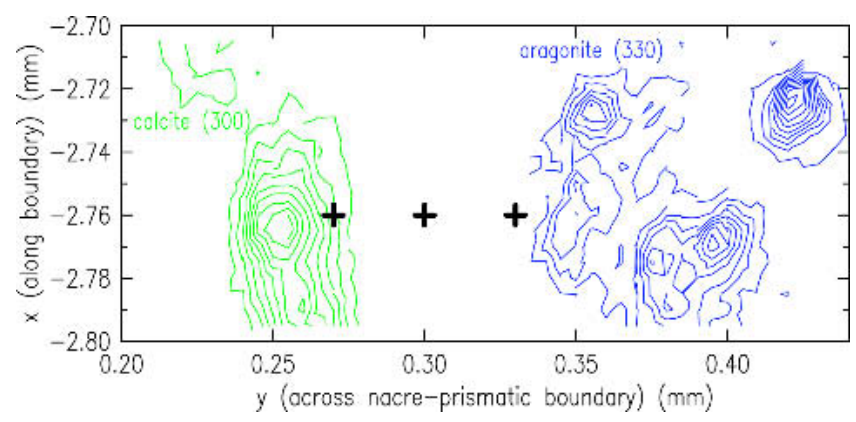

FIG. 3. Bragg peak intensity in the region of the nacre-prismatic boundary. Green contours: calcite (300) peak intensity. Blue contours: aragonite (330) intensity. Black crosses: locations at which two-theta scans were performed, as described in the text. 


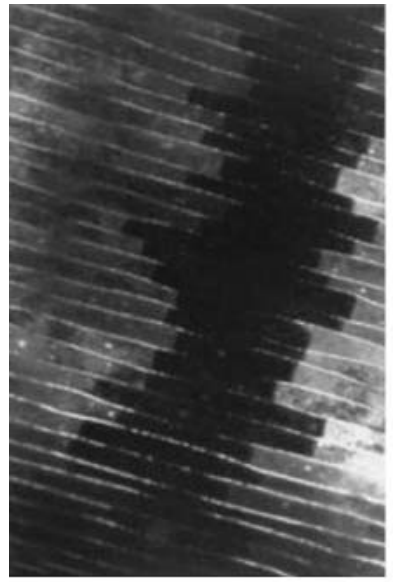

(a)

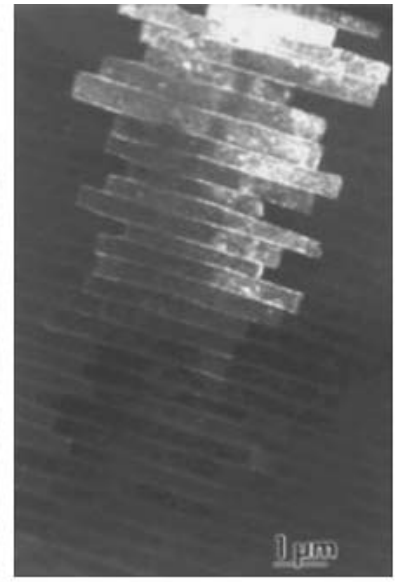

(b)

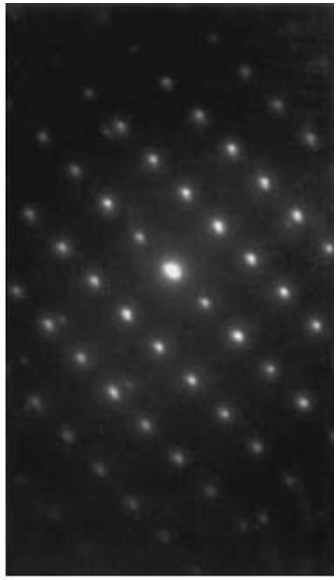

(c)

FIG. 4. (a) Bright-field image of red abalone nacre, showing nacre platelets in an end-on view. (b) Dark-field image highlighting the extent of platelets sharing the same crystallographic alignment in the $a-b$ plane. (c) Selected-area diffraction pattern corresponding to the image in (b).

alignment. Some research has suggested that new nacre layers are "seeded" in their orientations by crystal channels that grow through overlying layers of organic material, in a process that fixes the new layers' crystal orientation to match the tablet below it. ${ }^{4}$ Our $\mathrm{x}$-ray results show that this mechanism, if it exists, only persists over the span of a few dozen platelets.

Now we discuss the "empty space" between the contours of Fig. 3, where we were unable to find strong peaks along the surface normal. At the positions marked by black crosses in Fig. 3, we scanned the detector angle $2 \theta$. This is equivalent to scanning the sample in a powder diffraction mode. Three experimental scans are shown in Fig. 5. The top curve was taken at the position at the edge of the calcite grain $(y=0.17, x=-2.76$, the leftmost black cross in Fig. 3). At high $2 \theta$ values, off range of this plot, the calcite (300) and (301) are the only peaks observed. This curve therefore shows the noise level in the region of interest. The next two curves show peaks above the background, at places corresponding to nominal aragonite peak positions as marked. This is evidence for a jumble of aragonite grains in the $10-\mu \mathrm{m}$ illuminated spot. Based on the relative intensities of the peaks, the jumble is not a true powder. The computed aragonite powder spectrum is plotted at the bottom of the figure. For example, it is clear that the experimental and theoretical curves exhibit different intensities on the (220), (040), and (221) peaks. Because not all peaks observed have $(H K 0)$ indices, at least some crystallites do not lie with the $c$ axis within the boundary plane. Studies by transmission electron microscopy find a similar texture, with discrete crystals having random orientation relative to the ordered region far from the boundary (Sarikaya et al., manuscript in preparation). Further studies will be necessary to make stronger statements about the distributions of these grains near the calcite.

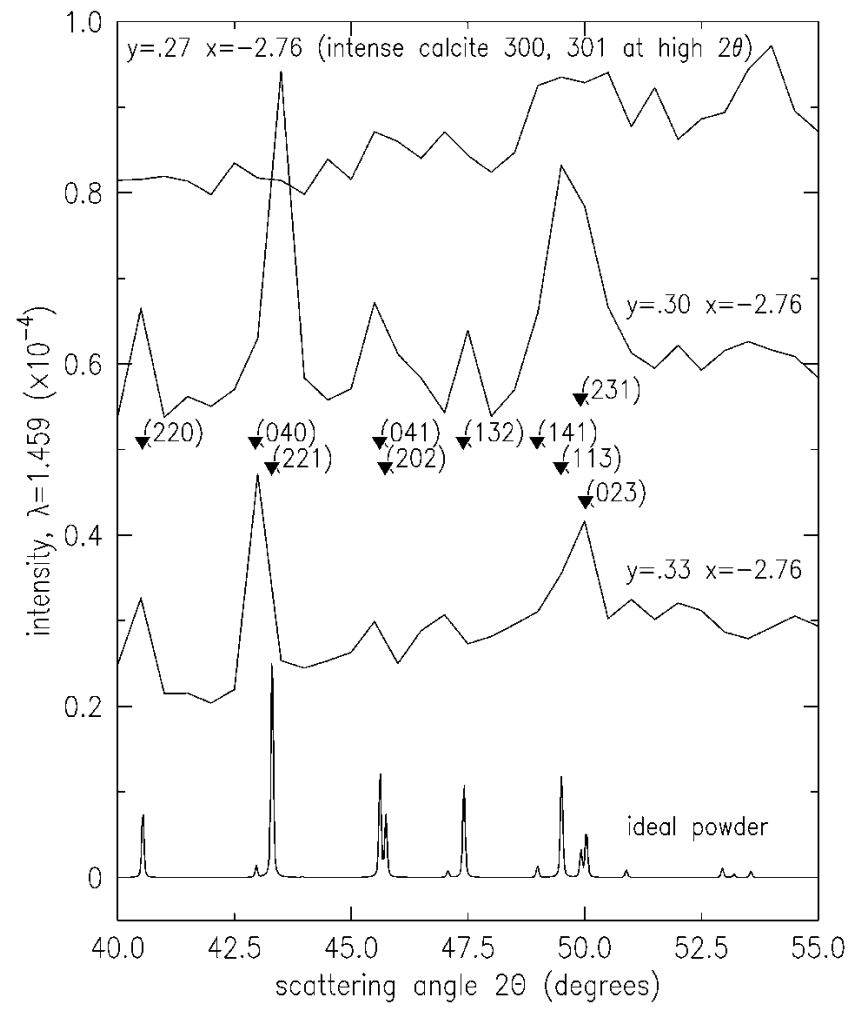

FIG. 5. Comparison of experimental and calculated aragonite diffraction patterns. Top curve corresponds to the first black cross in Fig. 3, within the calcite grain. Next two curves were taken at positions closer to the aragonite side of the boundary (middle and right black crosses in Fig. 3). These show peaks at aragonite positions as marked by arrows. The peak intensities for an ideal powder distribution of aragonite are shown by the spectrum at bottom.

\section{CONCLUSIONS}

Synchrotron x-ray microbeam diffraction measurements allow us to probe the texture and crystallography of biominerals on length scales that range from $10 \mu \mathrm{m}$ to 
several millimeters. At the nacre-prismatic boundary of red abalone, we observe millimeter-long calcite grains that impinge the boundary at $45^{\circ}$ angles, suggestive of nucleation on (104) planes and subsequent growth along the $c$ axis toward the nacre region. Within $100 \mu \mathrm{m}$ of the nacre-prismatic interface, calcite and aragonite crystals lose their bulk orientational order but do not attain an ideal powder texture. Columns of nacre tablets do not preserve their crystal alignment along the column for more than about $40 \mu \mathrm{m}$, suggesting that alignmentpreserving growth modes between successive layers of a column may not be the most important influence on morphology. Synchrotron x-ray microbeam studies, which combine micrometer-scale spatial sensitivity, highresolution diffraction data, and the capability to map texture on millimeter length scales, will play an important role in the study of biominerals and biomimetic materials in the future.

\section{ACKNOWLEDGMENTS}

The authors want to thank I.C. Noyan of IBM and J.L. Jordan-Sweet of the NSLS for assistance with the microdiffraction technique. M.S. thanks the Army Research Office (ARO) for support through the DURINT Program (Defense University Research Initiative on Nanotechnology) and AFOSR for grants under the Bio-Inspired Concepts Program. The National Synchrotron Light Source is supported under United States Department of Energy Contract No. DE-AC02-98CH10886.

\section{REFERENCES}

1. A.P. Jackson, J.F.V. Vincent, and R.M. Turner, The mechanical design of nacre, Proc. R. Soc. London B 234, 415 (1988).
2. M. Sarikaya and I.A. Aksay, Biomimetics: materials fabrication through biology, Biomimetics (American Institute of Physics, New York, 1992), pp. 34-85.

3. S. Manne, C.M. Zaremba, R. Giles, L. Huggins, D.A. Walters, A. Belcher, D.E. Morse, G.D. Stucky, J.M. Didymus, S. Mann, and P.K. Hansma, Atomic force microscopy of the nacreous layer in mollusc shells, Proc. R. Soc. London B 256, 17 (1994).

4. T.E. Schäffer, C. Ionescu-Zanetti, R. Proksch, M. Fritz, D.A. Walters, N. Almqvist, C.M. Zaremba, A.M. Belcher, B.L. Smith, G.D. Stucky, D.E. Morse, and P.K. Hansma, Does abalone nacre form by heteroepitaxial nucleation or by growth through mineral bridges? Chem. Mater. 9, 1731 (1997).

5. Q.L. Feng, F.Z. Cui, G. Pu, R.Z. Wang, and H.D. Li, Crystal orientation, toughening mechanisms and a mimic of nacre, Mater. Sci. Eng., C 11, 19 (2000).

6. T. Graham and M. Sarikaya, Growth dynamics of red abalone shell: a biomimetic model, Mater. Sci. Eng., C 11, 145 (2000).

7. E. Zolotoyabko and J.P. Quintana, Non-destructive microstructural analysis with depth resolution: Application to seashells, J. Appl. Crystallogr. 35, 594 (2002).

8. M. Fritz, A.M. Belcher, M. Radmacher, D.A. Walters, P.K. Hansma, G.D. Stucky, D.E. Morse, and S. Mann, Flat pearls from biofabrication of organized composites on inorganic substrates. Nature 371, 49 (1994).

9. C.M. Zaremba, A.M. Belcher, M. Fritz, Y. Li, S. Mann, P.K. Hansma, D.E. Morse, J.S. Speck, and G.D. Stucky, Critical transitions in the biofabrication of abalone shells and flat pearls, Chem. Mater. 8, 679 (1996).

10. A.M. Belcher, X.H. Wu, R.J. Christensen, P.K. Hansma, G.D. Stucky, and D.E. Morse, Control of crystal-phase switching and orientation by soluble mollusc-shell proteins, Nature 381, 56 (1996).

11. M. Sarikaya and I.A. Aksay, in Results and Problems in Cell Differentiation in Biopolymers, edited by S.T. Case, Nacre of abalone shell: a natural multifunctional nanolaminated ceramicpolymer composite material. (Springer, Amsterdam, 1992), pp. $1-25$.

12. I.C. Noyan, S.K. Kaldor, P-C. Wang, and J. Jordan-Sweet, A cost-effective method for minimizing the sphere-of-confusion error of x-ray microdiffractometers, Rev. Sci. Instrum. 70, 1300 (1999). 